\title{
Distributed Random Process for a large-scale Peer-to-Peer Lottery
}

\author{
Stéphane Grumbach and Robert Riemann \\ Inria \\ \{stephane.grumbach,robert.riemann\}@inria.fr
}

\begin{abstract}
Most online lotteries today fail to ensure the verifiability of the random process and rely on a trusted third party. This issue has received little attention since the emergence of distributed protocols like Bitcoin that demonstrated the potential of protocols with no trusted third party. We argue that the security requirements of online lotteries are similar to those of online voting, and propose a novel distributed online lottery protocol that applies techniques developed for voting applications to an existing lottery protocol. As a result, the protocol is scalable, provides efficient verification of the random process and does not rely on a trusted third party nor on assumptions of bounded computational resources. An early prototype confirms the feasibility of our approach.
\end{abstract}

Keywords: distributed aggregation, online lottery, DHT, trust, scalability

\section{Introduction}

Lottery is a multi-billion dollar industry [1]. In general, players buy lottery tickets from an authority. Using a random process, e.g. the drawing of lots, the winning tickets are determined and the corresponding ticket owners receive a reward.

In some lotteries, the reward may be considerable, and so is the incentive to cheat. The potential of fraud gained attention due to the Hot Lotto fraud scandal. In 2015, the former security director of the Multi-State Lottery Association in the US was convicted of rigging a 14.3 million USD drawing by the unauthorised deployment of a self-destructing malware manipulating the random process [2].

In order to ensure fair play and ultimately the trust of players, lotteries are subject to strict legal regulations and employ a technical procedure, the lottery protocol, to prevent fraud and convince players of the correctness. Ideally, players should not be required to trust the authority. Verifiable lottery protocols provide therefore evidence of the correctness of the random process.

In a simple paper-based lottery protocol, tickets are randomly drawn under public supervision of all players from an urn with all sold tickets to determine the winners. Afterwards, all tickets left over in the urn are also drawn to confirm their presence and convince the losers of the correctness. Without public supervision, the random process can be repeated until by chance a predefined result occurred. Further, the process can be replaced entirely by a deterministic process. 
In practice, the public supervision limits the number of lottery players and is further very inconvenient, because players are required to respect the time and locality of the drawing procedure. With the advent of public broadcasting channels, first newspapers, then radio and television broadcasting, protocols were employed that replaced the public supervision by a public announcement. Only few players and notaries verify here the correctness of the random process. In consequence, the majority of non-present players are required to trust the few present individuals for the sake of scalability. With the increasing availability of phones and later the internet, protocols have been adapted to allow also the remote purchase of lottery tickets, e.g. from home or a retail store.

The technical evolution lead to a gradual change of how people play lottery, but in many cases, the drawing procedure has not been adapted and resembles more a legacy that prevails for nostalgic reasons than to provide security. In the simple paper-based lottery protocol, the chain of custody establishes trust. All operations may be inspected by eye-sight.

This technique cannot be directly adapted for an online lottery. Thus, most verifiable online lottery protocols $[3,4,5]$ rely on a concept based on two elements. All players can actively contribute to the random process. Nobody can compute the random process result or its estimation as long as it is possible to contribute or buy new tickets. The latter is required to prevent educated contributions to circumvent the uniform distribution of the random process. It is the lottery protocol that must ensure the order in time of the contribution and the actual determination of winners.

A protocol consisting of equipotent players contributing each to the randomness of the publicly verifiable random process is promising for its similarity with the simple paper-based lottery protocol. Again, all players participate in the execution and supervision of the random process. The feasibility to construct such protocols with no trusted third parties has been demonstrated by the cryptocurrency Bitcoin [6] that is a distributed protocol for remote financial transactions, while previously online banking based on trusted financial authorities, the bank institutes and central banks, has been without alternative [7]. Lottery protocols based on Bitcoin have been already considered [8], c.f. Section 2.

Although different, the security requirements of lotteries share common concerns with those of voting systems [9]. Both lottery and voting protocols have to assure trust in an environment of mutual distrust among players, respectively voters, and the potentially biased authorities. The literature on voting protocols and online voting protocols is extensive and comprises flexible protocols that may be adapted to different voting systems beyond the general case of majority voting. Of particular interest for a lottery are online voting protocols that allow for a random choice. Already the paper-based voting common for general elections provides a solution to improve the scalability of the simple paper-based lottery protocol: the introduction of multiple offices run in parallel. We focus on online voting protocols that do not rely on trusted parties and aim to provide security properties that we adopt for lottery applications as follows: 
Correctness of the random process All numbers are equally likely to win. Nobody can predict the random process better than guessing.

Verifiability of the random process Players can be convinced that the random process has not been manipulated.

Privacy of the player Players do not learn the identity of other players to prevent blackmailing or begging.

Eligibility of the ticket Tickets cannot be forged. Especially, it is impossible to create a winning ticket after the outcome of the random process is known.

Confidentiality of the number Numbers are confidential to ensure fairness. Tickets of other players cannot be copied to reduce their potential reward.

Completeness of the reward Players can verify the number of sold tickets that may determine the reward.

Our contribution is a novel protocol for verifiable large-scale online lotteries with no trusted authority to carry out the random process, for which we use concepts originating from online voting.

The paper is organized as follows. In the next section, we review related work addressing both lottery and voting protocols. Our protocol is based on an existing online lottery protocol and the distributed hash table Kademlia that are presented in Section 3. Then, we introduce our protocol in Section 4 and discuss its properties in Section 5 .

\section{Related Work}

Different protocols have been proposed that allow players to contribute to the publicly verifiable random process and take measures to prevent early estimations of the result while it is still possible to contribute.

A trivial solution in the context of secure parameters for cryptography is recalled in [10]. In a first round, all players choose secretly a number and publish on a public broadcasting channel a commitment on their number, e.g. using a hash. In a second round, all secret numbers are revealed and verified using the commitment. Finally, all values are concatenated using the XOR operation to form the result. The protocol owes its correctness due to the clear separation in two rounds of player's contributions. However, the authors stress that the protocol is neither robust nor scalable. A termination is not possible if one player does not reveal its secret number and for the verification, all players have to run as many XOR operations and send as many messages as there are players.

Subsequently, a random process protocol with only one round is proposed [10]. A delay between player contribution and winner identification by the authority is imposed, so that estimations would be available only after contributions are no longer allowed. Players or any other third party can engage before a deadline in the collection of arbitrary data, e.g. using social networks like Twitter, to generate a seed, an essentially random bit string. Right after the deadline, the authority publishes a commitment on an additional, secretly chosen seed. Both seeds provide the input for a proof of work. A proof of work is computationally 
expensive to generate and thus time-consuming. A delay is inevitable. However, due to its asymmetry, the proof allows for efficient verification. Once the proof is found, the winners are derived from it. The additional seed prevents dishonest players to predict the results for different potential last-minute contributions. One has to assume that the last honest contribution is made sufficiently late to prevent the same attack from the authority.

Chow's online lottery protocol [5] published prior to [10] relies on a technique called delaying function [3] that is similar to a proof of work, but is not asymmetric and does not provide efficient verification. The authority commits here on the concatenation of the players' commitments on their secretly chosen number and derives then the winners. Players can claim the reward by publishing the input data of their commitment. Similar to [10], a late honest player commitment is assumed to prevent a prediction by the authority. Then, all security measures from the introduction are provided. The protocol requires players to process the commitment of all other players and recompute the delaying function in order to verify the random process, which is impractical for large-scale lotteries.

Solutions for a scalable probabilistic verification of online lotteries [11] or online voting [12] have been presented based on a concatenation/aggregation over a tree structure. In order to verify the result at the root of the tree, players or voters can repeat the computation of intermediate results for a predefined or random subset of all tree nodes. With increasing number of verified nodes, the probability of a manipulated result at the root node diminishes.

Other online lottery protocols introduce mutually distrusting, non-colluding authorities to allow for a separation of powers. In [13], a distinct auditor ensures secrecy and immutability of the player's tickets and prevents the lottery authority from adding illegitimately tickets. For this, blind signatures and public-key encryption are employed. The protocol does not cover the random process and its verification. Authorities are assumed not to collude.

In [14], the secrecy of online lottery and voting protocols is addressed at the same time. A mechanism based on homomorphic encryption, distributed key generation and threshold decryption is proposed. A set of mutually distrusting authorities have to cooperate to decrypt the result of the random process or the voting. A colluding set of dishonest authorities below the threshold cannot reveal prematurely the result, i.e. to add a winning ticket in the lottery case. Players or voters are entitled to trust that the set of dishonest, colluding authorities does not meet the threshold. Ideally, the power to decrypt would be shared among all players or voters. Practically, this is often not feasible due to scalability issues.

The Scalable and Secure Aggregation (SPP) online voting protocol [15] builds also on distributed decryption and employs a tree overlay network to improve the scalability. A small set of authorities is randomly chosen among all voters. If too many of those chosen voters are absent after the aggregation, the decryption threshold cannot be reached and, consequently, a protocol termination is impossible.

The potential of the Bitcoin blockchain [6] for a distributed random process has been examined. However, it has been shown that the manipulation of pre- 
sumably random bits is realistic even with limited computational capacity and financial resources [8]. An integration of the proof of work from [10] and an alternative crypto-currency Ethereum [16] has been proposed ${ }^{1}$ with no practical solution yet for a verification due to the limitation imposed by the blockchain.

\section{Preliminaries}

The starting point for the proposed protocol is the centralised online lottery protocol of Chow et al. [5], recalled hereafter with an alternative verification based on hash trees [11]. For the proposed lottery protocol, we choose to distribute the random process to all players. The overlay network comprising all players is provided by the distributed hash table (DHT) Kademlia [17] that is described in Section 3.2. The integration of these building blocks is shown in Section 4 .

\subsection{Centralised Online Lottery Protocol}

The following presentation of Chow's protocol $[5,11]$ is reduced to aspects required for our proposition. We use the following notation:



Chow's protocol implements a lottery in which every player $P_{i}$ has to choose a number $n_{i} \in \mathbb{L}$ and send a commitment on it to the authority $A$. $A$ aggregates all commitments to a value $h$. That means, every $P_{i}$ contributes to $h$. The aggregate $h$ is used as an input parameter for a delaying function (DF) preventing $A$ from early result estimations. The outcome of DF is used to compute the winning number $n_{R}$ with a verifiable random function and the secret key of $A$. Players do not have the secret key required to compute $n_{R}$, but can verify $n_{R}$ using the public key of $A$.

During the ticket purchase phase, $P_{i}$ acquires from $A$ a personal sequence number $s_{i} . P_{i}$ has to choose its number $n_{i}$ and a random bit string $r_{i}$ to compute its commitment ticket ${ }_{i}$ with bit string concatenation $\|$ and XOR operation $\underline{\vee}$. $P_{i}$ sends ticket ${ }_{i}$ to $A$ and receives in return the signature $\sigma_{A}\left(\right.$ ticket $\left._{i}\right)$ as a receipt.

$$
\text { ticket }_{i}=s_{i}\left\|\left(n_{i} \underline{\vee} \eta_{0}\left(r_{i}\right)\right)\right\| \eta\left(n_{i}\left\|s_{i}\right\| r_{i}\right)
$$

The DF cannot be evaluated before $h$ depending on all commitments is given, which is ideally only after the purchase phase. In [5], the DF input parameter

\footnotetext{
${ }^{1}$ http://www.quanta.im, https://kiboplatform.net (accessed 02/02/2017)
} 
$h$ is recursively computed from all $n$ commitments with $h=\eta\left(\operatorname{chain}_{n}\right)$ and chain $_{i}=\eta\left(\right.$ chain $_{i-1}||$ ticket $\left._{i}\right)$ with an empty initial chain chain 0 . An alternative introduced in [11] consists of a computation of $h$ using a $T$-ary Merkle tree [18] with ticket ${ }_{i}$ assigned to the leaf tree nodes. In both cases, all ticket ${ }_{i}$ are published to allow the verification of $h$ by the players requiring memory and computational resources of respectively $\mathcal{O}(n)$ and $\mathcal{O}\left(\log _{T}(n)\right)$.

Once the authority has published the verifiable winning number $n_{R}$, the reward claiming phase begins in which players $P_{i}$ with $n_{i}=n_{R}$ provide their sequence number $s_{i}$ and their secret random value $r_{i}$ to $A$ via a secure channel. Upon verification of the commitment ticket ${ }_{i}$ by $A$, the reward is granted. $P_{j}$ with $n_{j} \neq n_{R}$ may verify that their commitment ticket ${ }_{j}$ has been used to compute $h$ and are assumed to have trust in the infeasibility of $A$ to compute DF more than once between the latest honest ticket contribution and the publication of $n_{R}$.

\subsection{Distributed Hash Table Kademlia}

The distributed hash table (DHT) Kademlia [17] provides efficient discovery of lottery players and routing which is a precondition for the aggregation protocol in Section 4.1. Therefore, a binary overlay network is established in which each player $P_{i}$ is assigned to a leaf node $x_{i}$, that is a bit string of size $B$. The notation is as follows:

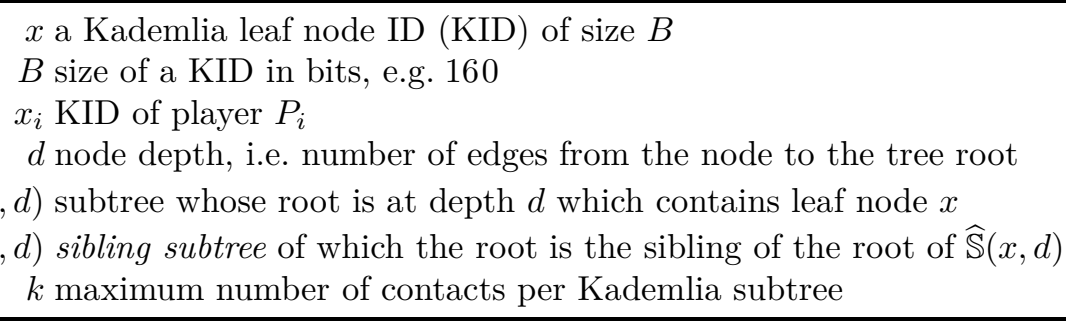

The leaf node identifiers $x \in\{0,1\}^{B}$ (B bits) span the Kademlia binary tree of height $B$ and are denoted KID. Each player $P_{i}$ joins the Kademlia overlay network using its KID defined as $x_{i}=\eta\left(t_{i}\right)$ with an authorization token $t_{i}$ and the hashing function $\eta(\cdot)$. The value $t_{i}$ is generated as part of the ticket purchase. $B$ is chosen sufficiently large, so that hash collisions leading to identical KIDs for distinct players are very unlikely. Consequently, the occupation of the binary tree is very sparse.

A node in the tree is identified by its depth $d \in\{0, \ldots, B\}$ and any of its descendant leaf nodes with KID $x$. A subtree $\widehat{\mathbb{S}}(x, d)$ is identified by the depth $d$ of its root node and any of its leaf nodes $x$. We overload the subtree notation to designate as well the set of players assigned to leaves of the corresponding subtree. Further, we introduce $\mathbb{S}(x, d)$ for the sibling subtree of $\widehat{\mathbb{S}}(x, d)$, so that $\widehat{\mathbb{S}}(x, d)=\widehat{\mathbb{S}}(x, d+1) \cup \mathbb{S}(x, d+1)$. The entire tree is denoted $\widehat{\mathbb{S}}(x, 0)$. We observe that $\forall d: P_{i} \in \widehat{\mathbb{S}}\left(x_{i}, d\right)$ and $\forall d: P_{i} \notin \mathbb{S}\left(x_{i}, d\right)$. 


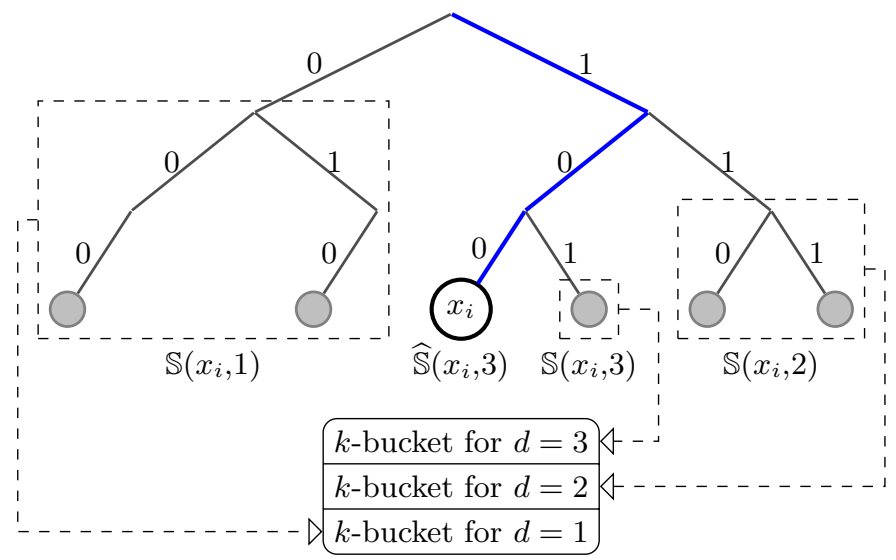

Fig. 1. Example of Kademlia $k$-buckets for KID $x_{i}=100$ assuming $B=3$. The sparse tree is partitioned into subtrees $\mathbb{S}\left(x_{i}, d\right)$ with their root node depth $d$. The $k$-buckets for each $d$ contain at most $k$ players $P_{j} \in \mathbb{S}\left(x_{i}, d\right)$.

Kademlia defines the distance $d\left(x_{i}, x_{j}\right)$ between two KIDs as their bit-wise XOR interpreted as an integer. In general, a player $P_{i}$ with KID $x_{i}$ stores information on players with $x_{j}$ that are close to $x_{i}$, i.e. for small $d\left(x_{i}, x_{j}\right)$. For this purpose, $P_{i}$ disposes of a set denoted $k$-bucket of at most $k$ players $P_{j} \in \mathbb{S}\left(x_{i}, d_{j}\right)$ for some $d_{j}>0 .{ }^{2}$ See Fig. 1 for an example. The size of subtrees decreases exponentially for growing depth $d$. Hence, the density of known players of corresponding $k$-buckets grows exponentially.

Kademlia ensures that the routing table, that is the set of all $k$-buckets, is populated by player lookup requests for random KIDs to the closest already known players. Requests are responded with a set of closest, known players from the routing table. One lookup might require multiple, consecutive requestresponse cycles. Further, Kademlia provides requests to lookup and store values. All operations scale with $\mathcal{O}(\log n)$ [19]. Kademlia is used by many BitTorrent clients and as such well tested.

\section{Distributed Lottery}

We introduce now the lottery protocol. It is run by an authority that handles the ticket purchase and carries out the distribution of the reward upon winner verification, but not the random process itself. The random process is distributed to all players using the protocol described below. The description of the lottery protocol is given in Section 4.2.

\footnotetext{
${ }^{2}$ Note that originally [17] the common prefix length $b$ is used to index $k$-buckets/sibling subtrees while we use the depth $d=b+1$ of the root of the subtree.
} 


\subsection{Distributed Aggregation Protocol}

We present a distributed aggregation protocol based on Kademlia. It relies on ADVOKAT [20], whose aggregation algebra, distributed aggregation algorithm, and measures to increase its Byzantine fault tolerance are briefly recalled.

Aggregation Algebra Aggregates are values to be aggregated, whether initial aggregates, constituting inputs from players, or intermediate aggregates obtained during the computation. The aggregation operation, $\oplus$, combines two child aggregates to a parent aggregate in $\mathbb{A}$, the set of aggregates. We assume $\oplus$ to be commutative. For the lottery, $\oplus$ maps pairwise bit strings provided by all players to one final bit string used to determine the winners. The algebra is sufficiently flexible to cover a broad range of aggregation-based applications and has been devised initially for distributed online voting [20].

Aggregates are manipulated through aggregate containers, i.e. a data structure that contains next to the aggregate itself the context of the ongoing computation. The aggregate container of an aggregate $a$ associates $a$ to a Kademlia subtree $\widehat{\mathbb{S}}(x, d)$ and ensures integrity and verifiability of the aggregation. It has the following attributes:



Similar to the aggregation of aggregates, one or two aggregate containers of $a_{1}$, $a_{2}$ can be aggregated to a parent aggregate container. To inherit the commutativity of the aggregation of aggregates $\oplus,\left(h_{1}, c_{1}\right)$ and $\left(h_{2}, c_{2}\right)$ must be sorted in e.g. ascending order of the child hashes $h_{1}$ and $h_{2}$.

Distributed Aggregation Algorithm Using the aggregation operator $\oplus$, every player $P_{i}$ computes the intermediate aggregate for all the parent nodes from its corresponding leaf node $x_{i}$ up to the root node of the Kademlia overlay tree. The aggregates used to compute any intermediate aggregate of a given subtree $\widehat{\mathbb{S}}\left(x_{i}, d\right)$ are given by its child nodes' aggregates of $\widehat{\mathbb{S}}\left(x_{i}, d+1\right)$ and $\mathbb{S}\left(x_{i}, d+1\right)$. Hence, aggregates have to be exchanged between players of the sibling subtrees and Kademlia's $k$-buckets provide the required contact information.

The aggregation is carried out in $B$ epochs, one tree level at a time. Epochs are loosely synchronized, because players may have to wait for intermediate aggregates to be computed in order to continue. First, every player $P_{i}$ computes a container for its initial aggregate $a_{i}$. The container is assigned to represent the subtree $\widehat{\mathbb{S}}\left(x_{i}, B\right)$ with only $P_{i}$. In each epoch for $d=B, \ldots, 1$, every player $P_{i}$ requests from a random $P_{j} \in \mathbb{S}\left(x_{i}, d\right)$ the aggregate container of subtree $\mathbb{S}\left(x_{i}, d\right)$. 
With the received container of $\mathbb{S}\left(x_{i}, d\right)$ and the previously obtained of $\widehat{\mathbb{S}}\left(x_{i}, d\right)$, player $P_{i}$ computes the parent aggregate container, that is then assigned to the parent subtree $\widehat{\mathbb{S}}\left(x_{i}, d-1\right)$. If $\mathbb{S}(x, d)=\emptyset$ for any $d$, the container of $\widehat{\mathbb{S}}(x, d-1)$ is computed only with the aggregate container of $\widehat{\mathbb{S}}(x, d)$ from the previous epoch.

After $B$ consecutive epochs, player $P_{i}$ has computed the root aggregate $a_{R}$ of the entire tree $\widehat{\mathbb{S}}\left(x_{i}, 0\right)$ that contains the initial aggregates of all players. If all players are honest, the root aggregate is complete and correct. Due to the commutativity of the container computation, all players find the same hash $h_{R}$ for the container of the root aggregate $a_{R}$. An individual verification is implicitly given, because every player computes $a_{R}$ starting with its $a_{i}$.

Byzantine Fault-Tolerance The distributed aggregation is very vulnerable to aggregate corruptions leading to erroneous root aggregates containers. We present intermediate results to safeguard the aggregation. Please refer to [20] for a more in-depth discussion. For the attack model, we assume a minority of dishonest (Byzantine) players controlled by one adversary that aims to interrupt the aggregation, and manipulate root aggregates. Dishonest players can behave arbitrarily. Like in Kademlia, time-outs are used to counter unresponsive players.

To prevent Sybil attacks, it must be ensured that a player a) cannot choose on its own discretion its tree position given by the leaf node $x_{i}$ but $\mathrm{b}$ ) can proof its attribution to $x_{i}$ [21]. Every player $P_{i}$ generates a key pair $\left(p k_{i}, s k_{i}\right)$ which must be signed by $A$. Hence, $P_{i}$ sends $p k_{i}$ to $A$ during the ticket purchase and receives the signature of $A$ to be used as the authorization token $t_{i}=\sigma_{A}\left(p k_{i}\right)$. The KID $x_{i}=\eta\left(t_{i}\right)$ is derived from $t_{i}$ and is neither chosen unilaterally by $A$ nor by $P_{i}$. Eventually, players provide for every message $m$ exchanged among players the signature of the sender $\sigma_{i}(m)$, its public key $p k_{i}$ to verify $\sigma_{i}(m)$, and the authorization token $t_{i}$ to verify $p k_{i}$.

Moreover, a dishonest authority shall be prevented to add new players after the aggregation has started and dishonest players to delay their contributions after predefined, global deadlines. In order to suppress both, signatures of those players are considered invalid, who are at the start of the aggregation not in the corresponding $k$-bucket even though the bucket contains less than $k$ players and should be exhaustive.

Further, player signatures are employed to detect deviations from the protocol. For every computed aggregate container of $\widehat{\mathbb{S}}\left(x_{i}, d\right)$ with hash $h$ and counter $c$, player $P_{i}$ produces an aggregate container signature $\sigma_{i}(h, d, c)$. Other players can verify the signature using $p k_{i}$ and verify using $x_{i}=\eta\left(t_{i}\right)$ that $P_{i} \in \widehat{\mathbb{S}}\left(x_{i}, d\right)$. Hence, $p k_{i}$ and $t_{i}$ must be provided along every signature $\sigma_{i}(h, d, c)$.

The impact of dishonest players is limited by redundant requests to confirm a computed so-called candidate container using signatures of other players on the same container hash as depicted in Fig. 2. Next to the proper signature (2) on $h$ and $h_{1}$, a signature on $h$ from a player in each child subtree (3) and (4)) and one on $h_{2}$ (1) must be provided for a confirmation if the respective subtree is non-empty which can be determined using Kademlia lookup requests. The number of distinct signatures on $h$, here 3 , is a security parameter denoted $l$. 


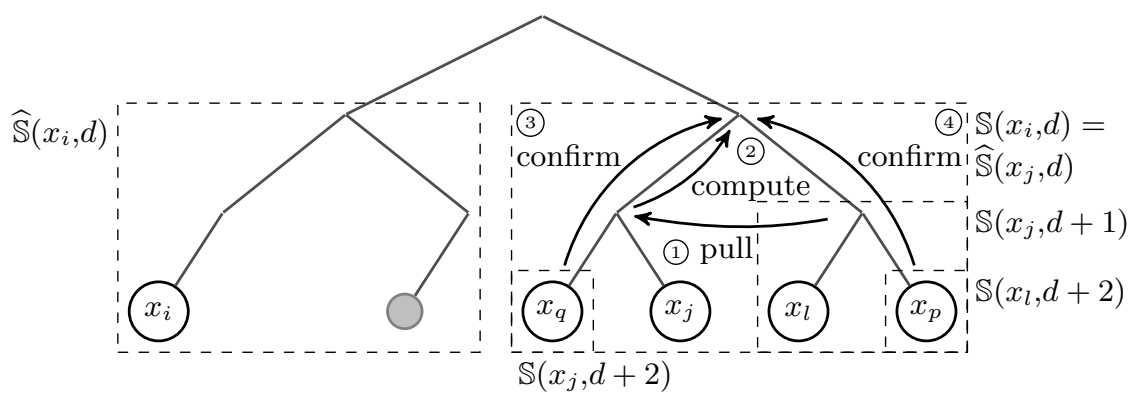

Fig. 2. $P_{j}$ with $x_{j}$ produces a confirmed aggregate container of $\mathbb{S}\left(x_{i}, b\right)$. This scheme applies to all tree levels with possibly large subtrees to request from. If the subtrees $\mathbb{S}\left(x_{j}, d+2\right), \mathbb{S}\left(x_{l}, d+2\right)$ are empty, the depth is further increased until a non-empty subtree may be found.

Only the confirmed container including the signatures is used to respond to requests from players in the sibling subtree. If a confirmation is not possible, e.g. due to non-cooperating dishonest players, the confirmed child containers are provided instead, so that the receiver can compute the aggregation on its own.

If confirmation requests reveal diverging containers, a majority vote using the number of distinct signatures for every container hash is used. If another container than the previously computed is selected and if $h_{2}$ differs, then the request for the sibling aggregate container (1) is repeated, otherwise, the previous epoch is repeated allowing for a recursive correction.

The majority vote confirms for subtrees with many players with great probability the aggregate container of the honest players. The attribution of KIDs $x_{i}$ to players $P_{i}$ is random, so that a global minority of dishonest players is uniformly distributed over all subtrees and a honest majority can be assumed for most local subtrees.

Though, dishonest players may have a local majority in subtrees with only few players. Here, an analysis of the signatures of confirmed containers allows honest players to detect dishonest behaviour in the following cases with certainty. Given two signatures $\sigma_{i}(h, d, c)$ and $\sigma_{i}\left(h^{\prime}, d, c\right)$ from the same player $P_{i}$ with different hashes $h \neq h^{\prime}, P_{i}$ deviated from the protocol with certainty if $c \leq l$. Either $P_{i}$ signed two distinct initial aggregate containers or accepted a non-confirmed container. For $c>l$, there is a non-zero probability that $P_{i}$ is honest, but may have been tricked. A manipulation may not be detected or only later during the recursive correction. The number of distinct signatures $l$ can be increased to detect manipulations with certainty for higher $c$, and may depend on the player configuration in the respective subtree.

At last, the root aggregate container $a_{R}$ shall be confirmed more often, i.e. more signatures on its hash $h$ are gathered from different players, to increase the confidence that it has been adopted by the majority of honest players. 


\subsection{Lottery Protocol}

The proposed protocol allows for a lottery with playing mode CL or LO [13]:

\section{Classic Lottery (CL)}

Rewards are distributed with respect to a randomly ordered list of all players.

\section{Lotto (LO)}

Rewards are distributed based on the secret, prior choice of each player.

The protocol has six phases of which ticket purchase, reward claiming and winner verification follow closely Chow's protocol [5]. Its model provides for an authority $A$, a tracker and players $P_{i}$. For CL, $\eta_{0}(\cdot)$ is identical to $\eta(\cdot)$, so that the root aggregate $a_{R}$ of the distributed aggregation is in the domain of the KIDs $x$.

\section{Setup}

1. A generates a key-pair $\left(p k_{A}, s k_{A}\right)$ and chooses a random bit string $r_{A}$.

2. A publishes the ticket purchase deadline, $p k_{A}, \eta(\cdot), \eta_{0}(\cdot)$ and $\eta\left(\eta\left(r_{A}\right)\right)$. Further, $A$ specifies the duration of the aggregation epoch for each tree level.

\section{Ticket Purchase}

1. $P_{i}$ picks a random string $r_{i}$, and for CL its number $n_{i}$. It generates $\left(p k_{i}, s k_{i}\right)$.

2. $P_{i}$ sends $p k_{i}$ to the authority and obtains in return a sequence number $s_{i}$ and its authorization token $t_{i}=\sigma_{A}\left(p k_{i}\right)$.

3. $P_{i}$ computes $x_{i}=\eta\left(t_{i}\right)$ and connects to the Kademlia DHT using an already connected contact provided by the authority or a separate tracker.

4. $P_{i}$ prepares its initial aggregate $a_{i}=\eta\left(\right.$ ticket $\left._{i}\right)$. For CL, ticket ${ }_{i}=s_{i} \| r_{i}$ and for LO, ticket ${ }_{i}=s_{i}\left\|\left(n_{i} \underline{\vee} \eta_{0}\left(r_{i}\right)\right)\right\| \eta\left(n_{i}\left\|s_{i}\right\| r_{i}\right)$, c.f. Section 3.1.

\section{Distributed Random Process}

1. After the ticket purchase deadline, $A$ publishes the number of sold tickets $n$.

2. All $P_{i}$ compute jointly the root aggregate $a_{R}$. The $\oplus$-operation is given by $a_{i} \oplus a_{j}=\eta\left(a_{i j}\right)$ with $a_{i j}=a_{i} \| a_{j}$, if $a_{i}<a_{j}$, otherwise $a_{i j}=a_{j} \| a_{i}$. It is a commutative variant of the binary Merkle tree scheme proposed in [11].

3. Proofs of protocol deviation in form of pairs of signatures are sent to $A$ that can reveal the corresponding players and revoke their right to claim a reward.

\section{Winner Identification}

1. A requests the root aggregate of multiple random $P_{i}$ until a considerably large majority of the sample confirmed one $a_{R}$.

2. $A$ publishes $a_{R}, r_{A}$ and the winning number $n_{w}=\eta_{0}\left(a_{R}\right) \underline{\vee} \eta_{0}\left(r_{A}\right)$.

3. For CL, $A$ computes all $x_{i}=\eta\left(t_{i}\right)$, orders all $P_{i}$ by their Kademlia XOR distance $d\left(n_{w}, x_{i}\right)=n_{w} \underline{\vee} x_{i}$ and players on a par by $n_{w} \underline{\vee} s_{i}$, and publishes as many ordered $x_{i}$ as there are rewards. For LO, winners $P_{i}$ have $n_{i}=n_{w}$. 


\section{Reward Claiming}

1. The winner $P_{i}$ sends all its confirmed aggregate containers to $A$ to proof their participation. For LO, $P_{i}$ must also provide its ticket ${ }_{i}$ and $\left(s_{i}, r_{i}\right)$.

2. Proofs are published for verification by other players.

\section{Winner Verification}

1. $\eta\left(\eta\left(r_{A}\right)\right)$ is computed for comparison with the previously published value and $n_{w}$ is verified.

2. Players verify that winner $P_{i}$ participated in the aggregation by comparing its published containers with their computed containers.

3. For CL, player verify the order of the published winners and compare it to their own positioning. For LO, ticket ${ }_{i}$ is reproduced for the given $\left(s_{i}, r_{i}\right)$ and its hash must equal $a_{i}$ found in the published confirmed aggregate containers.

4. If the rewards depend on the number of sold tickets $n, n$ is compared to the counter $c$ of the root aggregate container.

\section{Evaluation}

We analyse the protocol with respect to the security properties introduced in Section 1 under the adversary model from Section 4.1 of an adversary $D$ controlling a fraction $b<0.5$ of dishonest players of $n$ players in total. The performance of the protocol depends upon $b$, the security parameter $l$ and the distribution of honest and dishonest players over the tree. We assume that $D$ and $A$ collude.

Most Likely Scenario Due to the uniform player distribution and for a reasonably sized $b, D$ has most likely a dishonest majority only in subtrees with large depth $d>1$ containing only a small number $n^{\prime}$ of players. $l$ can be adjusted to detect container manipulations of subtrees with $n^{\prime} \leq l$ using signatures. Most likely, all dishonest players have to provide a container with their signature to at least one honest player, which corresponds to a commitment to their ticket $_{i}$, before $D$ can learn all containers for a given depth $d$.

1. The correctness of the random process and its implicit verification [11] due to the distributed computation is with great probability ensured, because $D$ cannot change or add tickets after a prediction becomes possible.

2. The privacy of players is ensured. Other players cannot learn the identity of each other from the exchanged messages. ${ }^{3}$

3. The authorization token $t_{i}$ ensures eligibility. A participation after the aggregation has started even with a valid $t_{i}$ is unlikely, because honest players close in the tree deny belated players and do not confirm their containers.

4. The commitment scheme for LO provides confidentiality, because number $n_{i}$ of $P_{i}$ cannot be revealed without knowledge of the secret $r_{i}$ [5].

5 . The counter $c$ of the root aggregate container allows to examine the completeness of the reward.

\footnotetext{
${ }^{3}$ The leak of the identity due to the communication channel, e.g. by the IP address,
} may be solved using privacy networks like Tor and is out of the scope of this paper. 
Worst Case Scenario The distribution of honest and dishonest players is highly unbalanced. We assume a majority of dishonest players in a subtree $\widehat{\mathbb{S}}\left(x_{e}, d\right)$ for some $d$ with $n^{\prime}>l$. Neither the majority nor the confirmation criterion prevent a manipulation with certainty. The local minority of honest players may be excluded from the aggregation unable to proof their participation. As the manipulation is bounded locally, correctness and eligibility are only locally violated.

If $D$ has further in all other non-sibling subtrees $\widehat{\mathbb{S}}(\cdot, d)$ at least one dishonest player to provide the local aggregate container, $D$ can compute with the secret $r_{A}$ from $A$ the winning number $n_{w}$ while the container for $\widehat{\mathbb{S}}\left(x_{e}, d\right)$ is not yet known to honest players and may be altered to change $n_{w}$. A proof of work is required to choose a particular $n_{w}$. The correctness is not ensured.

The distribution of $n^{\prime}$ honest or dishonest players to $\widehat{\mathbb{S}}\left(x_{e}, d\right)$ and its sibling subtree $\mathbb{S}\left(x_{e}, d\right)$ follows the Binomial distribution $B\left(n^{\prime}, p\right)$ with $p=0.5$ and a variance of the ratio of players in $\widehat{\mathbb{S}}\left(x_{e}, d\right)$ of $p^{2} / n^{\prime}$. As a result, the probability of a local dishonest majority decreases reciprocally in $n^{\prime} . n^{\prime}$ decreases for increasing $d$, but for large $d$, it is unlikely to have a dishonest player in all non-sibling subtrees for the limited number of dishonest players.

Scalability Kademlia's communication and memory resources are $\mathcal{O}(\log n)$ [19]. The same applies to the distributed aggregation and its verification [20] if upper bounds are defined for the number of attempts and stored container candidates of the confirmation and correction mechanism of the distributed aggregation.

\section{Conclusion}

We have presented a novel online lottery protocol that relies on a distributed random process carried out by all players in a peer-to-peer manner. Players are assumed to participate throughout the random process. Unlike Chow's protocol [5], it allows for both classic lottery and lotto. It provides correctness and verification of the random process based on the assumption of a well-distributed minority of dishonest players. In the most likely scenario, the correctness of the random process is based on an information theoretical secure sharing scheme instead of assumptions on the communication or computational capacities of the authority or the adversary. Further, cryptography has been reduced to asymmetric encryption and signatures. As in many distributed protocols $[6,15]$, the provided security is probabilistic, which may be acceptable for a lottery. We leave for future work a quantitative analysis of the impact of the adversary.

A basic demonstrator has been implemented to carry out a classical lottery. The authority has been omitted in favour of free participation. Redundant requests for Byzantine fault-tolerance are not covered yet. Based on HTML5, it runs in the browser. The implementation of ADAvOKAT is based on the Kademlia library $\mathrm{kad}^{4}$ and was tested previously with up to 1000 simulated nodes [20]. Message passing among players relies on WebRTC allowing for browser-tobrowser communication. Tests have been run with few players at this stage.

\footnotetext{
${ }^{4}$ http://kadtools.github.io/, v1.6.2 released on November 29, 2016
} 
Acknowledgments The authors would like to thank Pascal Lafourcade and Matthieu Giraud for fruitful discussions concerning the security of the lottery protocol and the underlying distributed aggregation algorithm.

\section{References}

1. Isidore, C.: Americans spend more on the lottery than on ... (2015). http://money.cnn.com/2015/02/11/news/co (visited on 16/02/2017)

2. Rodgers, G.: Guilty verdict in Hot Lotto scam, but game safe, official says, (2015). http://dmreg.co/1JbGgRN (visited on 23/01/2017)

3. Goldschlag, D.M., and Stubblebine, S.G.: Publicly verifiable lotteries: Applications of delaying functions. In: Proc. Financial Crypt. 1998, pp. 214-226. Springer (1998)

4. Zhou, J., and Tan, C.: Playing Lottery on the Internet. In: Proc. ICICS 2001, pp. 189-201. Springer (2001)

5. Chow, S.S.M., Hui, L.C.K., Yiu, S.M., and Chow, K.P.: An e-Lottery Scheme Using Verifiable Random Function. In: Proc. ICCSA 2005, pp. 651-660. Springer (2005)

6. Nakamoto, S.: Bitcoin: A Peer-to-Peer Electronic Cash System, (2008). https://bitcoin.org/bitcoin.pdf

7. Perez-Marco, R.: Bitcoin and Decentralized Trust Protocols. (2016)

8. Pierrot, C., and Wesolowski, B.: Malleability of the blockchain's entropy. In: ArcticCrypt 2016, pp. 1-20

9. Lambrinoudakis, C., Gritzalis, D., Tsoumas, V., Karyda, M., and Ikonomopoulos, S.: Secure electronic voting: The current landscape. In: Secure Electronic voting, pp. 101-122. Springer, USA(2003)

10. Lenstra, A.K., and Wesolowski, B.: A random zoo: sloth, unicorn, and trx. NIST Workshop on Elliptic Curve Cryptography Standards 3 (2015)

11. Liu, Y., Hu, L., and Liu, H.: Using an Efficient Hash Chain and Delaying Function to Improve an e-Lottery Scheme. Int. J. Comput. Math. 84(7), 967-970 (2007)

12. Markowitch, O., and Dossogne, J.: E-voting : Individual verifiability of public boards made more achievable. In: 31. Symp. on Information Theory in the Benelux, pp. 5-10. Werkgemeenschap voor Informatie en Communicatietheorie (2010)

13. Kuacharoen, P.: Design and Implementation of a Secure Online Lottery System. In: Proc. IAIT 2012. Pp. 94-105. Springer Berlin Heidelberg(2012)

14. Fouque, P.-A., Poupard, G., and Stern, J.: Sharing Decryption in the Context of Voting or Lotteries. In: Proc. Financial Crypt. 2000, pp. 90-104. Springer (2001)

15. Gambs, S., Guerraoui, R., Harkous, H., Huc, F., and Kermarrec, A.-M.: Scalable and Secure Aggregation in Distributed Networks. arXiv e-prints (2011)

16. Wood, G.: Ethereum: A Secure Decentralised Generalised Transaction Ledger, (2014). http://gavwood.com/paper.pdf

17. Maymounkov, P., and Mazieres, D.: Kademlia: A Peer-to-Peer Information System Based on the XOR Metric. Springer Berlin Heidelberg, Berlin, Heidelberg (2002)

18. Merkle, R.C.: A Digital Signature Based on a Conventional Encryption Function. In: Proc. CRYPTO 1987, pp. 369-378. Springer Berlin Heidelberg (1988)

19. Cai, X.S., and Devroye, L.: A probabilistic analysis of Kademlia networks. In: Proc. ISAAC 2013, pp. 711-721 (2013)

20. Riemann, R., and Grumbach, S.: Secure and Trustable Distributed Aggregation based on Kademlia. In: Proc. 32nd IFIP SEC, pp. 171-185. Springer, Rome (2017) 
21. Baumgart, I., and Mies, S.: S/Kademlia: A practicable approach towards secure key-based routing. In: Proc. ICPADS '07, pp. 1-8. ICS, USA (2007) 